\title{
Sulfamethazine Bisulfate
}

National Cancer Institute

\section{Source}

National Cancer Institute. Sulfamethazine Bisulfate. NCI Thesaurus. Code C87217.

A bisulfate salt form of sulfamethazine, a sulfonamide antibiotic used in the lifestock industry. 